\title{
Feedback Stabilization of Relative Equilibria for Mechanical Systems with Symmetry
}

\author{
Anthony M. Bloch *, \\ Jerrold E. Marsden ${ }^{\dagger}$ \\ and \\ Gloria Sánchez de Alvarez ${ }^{\ddagger}$
}

\begin{abstract}
This paper is an outgrowth of the work of Bloch, Krishnaprasad, Marsden and Sánchez de Alvarez [1992], where a feedback control that stabilizes intermediate axis rigid body rotation using an internal rotor was found. Stabilization is determined by use of the energy-Casimir (Arnold) method. In the present paper we show that this feedback controlled system can be written as the Euler-Lagrange equations for a modified Lagrangian: a velocity shift associated with a change of connection turns the free (unforced) equations into the feedback controlled equations. We also show how stabilization of the inverted pendulum on a cart can be achieved in an analogous way. We provide a general systematic construction of such controlled Lagrangians.

The basic idea is to modify the kinetic energy of the free Lagrangian using a generalization of the Kaluza-Klein construction in such a way that the extra terms obtained in the Euler-Lagrange equations can be identified with control forces. The fact that the controlled system is Lagrangian by construction enables one to make use of energy techniques for a stability analysis. Once stabilization is achieved in a mechanical context, one can establish asymptotic stabilization by the further addition of dissipative controls. The methods here can be combined with symmetry breaking controls obtained by modifying the potential energy and also can be used for tracking.
\end{abstract}

* Research partially supported by the National Science Foundation PYI grant DMS91-57556, AFOSR grant F49620-96-1-0100 and by a Guggenheim Fellowship.

${ }^{\dagger}$ Research partially supported by the National Science Foundation under Grant DMS9302992 and the Department of Energy under Contract DE-FG0395-ER25251.

${ }^{\ddagger}$ Research partially supported by CDCHT-ULA and CONICIT-Venezuela 


\section{Introduction}

The Motivating System: The Rigid Body with Rotors. In this paper we construct a class of stabilizing feedback control laws which generalize those introduced in Bloch, Krishnaprasad, Marsden and Sánchez de Alvarez [1992]. In that paper, a rigid body with a feedback controlled rotor is considered and motion near the unstable middle axis rotation of the rigid body is studied. It was shown that when a gain parameter $k$ exceeds a certain explicitly determined critical value, the motion is nonlinearly stabilized and this feedback stabilization can be understood within the context of the energy-Casimir (or Arnold) method for stability analysis. In addition, phase drifts caused by this feedback (and ultimately due to the symmetry in the problem) were studied.

Controlled Lagrangians. In the present paper we show that the specific feedback law constructed for the rigid body with a rotor can be understood in terms of a general construction involving what we call controlled Lagrangians. In the example, one finds that the controlled Euler-Lagrange equations are identical with the Euler-Lagrange equations for the controlled Lagrangian. Moreover, we show that the construction of controlled Lagrangians has interesting geometric underpinnings, which are related to the Kaluza-Klein construction. We are using Kaluza-Klein theory in a very elementary way in this paper; we mean it in the sense that, for example, the motion of a charged particle in a magnetic field can be viewed as geodesic motion for an appropriately constructed metric (see, for example, Marsden and Ratiu [1994] for an exposition).

The main purpose of this paper is to present the construction of a general class of controlled Lagrangians and to show how to apply it to two examples. The first example is rather simple, namely the problem of stabilization of an inverted pendulum on a cart. The second one is the motivating one of a rigid body with an internal rotor that was mentioned above.

In this paper we confine our attention to constructing a class of controlled Lagrangians that are obtained by modifying the kinetic energy of the given Lagrangian (assumed to be of the form kinetic minus potential energy). We do this by changing the underlying metric structure of the kinetic energy. It may also be viewed as a change of connection. A change of connection acts in much the same way as a change to a rotating frame and it generates new forces, the most interesting of which are Coriolis-type forces which can be stabilizing. In any event, our approach is designed to 
produce controllers that, by construction, will be associated with a new Lagrangian, and hence a Hamiltonian, system.

Advantages of the Present Approach. Some advantages over other possible feedback stabilization control strategies are as follows. First of all, it allows one to understand the stabilization in terms of energetics. Roughly speaking, a saddle point, for example, of the energy can be turned into a maximum or a minimum (this is not literally true since one has to take the symmetry group into account). Within this context, our method automatically constructs a Liapunov function for the control system and this provides a systematic method for determining when the control forces are stabilizing. Secondly, even though work is done by the control forces, there is an extension of the mechanical energy of the system that is conserved; one can think of it as a combined energy available to the mechanism and the control forces. This guarantees that, for example, the control rotors will never need to attain large velocities to achieve stabilization.

Related Approaches. Energy methods in control and stabilization have been used by many previous authors. The paper of Wang and Krishnaprasad [1992] uses gyroscopic forces in the context of the energy momentum method with applications to stabilizing controllers. This approach should be very useful in conjunction with our construction of controlled Lagrangians for the assessement of the stability of the associated feedback laws. Other references that were useful for us were Koditschek [1989], Koditschek and Rimon [1990] and Ballieul [1993] (and related references). Stabilization by feedback is just one control objective for which we expect these ideas to be useful. For example, we plan, in a forthcoming publication, to combine the ideas here with those of Leonard [1996] on symmetry breaking potentials, which will extend the method here from stabilization in the "internal balance variables" to stabilization in the symmetry directions (see also Leonard [1995] and Leonard and Marsden [1996]). We also plan to consider problems of tracking using related ideas.

\section{Bundles and Controlled Lagrangians}

Principal Connections. Let $G$ be a Lie group that acts freely and properly on the left on a configuration manifold $Q$ and let $S=Q / G$ be the corresponding shape space. Thus, the canonical projection $\pi: Q \rightarrow S$ defines a principal $G$-bundle. 
Recall that a principal connection $A$ on $Q$ is a $\mathfrak{g}$-valued 1-form $A$ : $T Q \rightarrow \mathfrak{g}$ such that $A\left(\xi_{Q}(q)\right)=\xi$ for each $\xi \in \mathfrak{g}$, where $\xi_{Q}$ is the infinitesimal generator of $\xi$ on $Q$ and secondly, $A$ is equivariant with respect to the action of $G$ on $T Q$ and the adjoint action of $G$ on $\mathfrak{g}$.

The vertical space is defined by

$$
\operatorname{Ver}_{q} Q=\left\{v_{q} \in T_{q} Q \mid T_{q} \pi\left(v_{q}\right)=0\right\},
$$

so that vertical tangent vectors are tangent to the fibers $\pi^{-1}(q)$. Equivalently,

$$
\operatorname{Ver}_{q} Q=\left\{\xi_{Q}(q) \mid \xi \in \mathfrak{g}\right\} .
$$

The horizontal space is

$$
\operatorname{Hor}_{q} Q=\left\{v_{q} \in T_{q} Q \mid A\left(v_{q}\right)=0\right\} .
$$

One can decompose every tangent vector $v_{q} \in T_{q} Q$ uniquely into a horizontal and vertical part relative to a given connection; we write

$$
T_{q} Q=\operatorname{Ver}_{q} \oplus \operatorname{Hor}_{q},
$$

where $\operatorname{Ver} v_{q}=\left(A\left(v_{q}\right)\right)_{Q}(q)$ and Hor $v_{q}=v_{q}-\operatorname{Ver} v_{q}$.

Kaluza-Klein Lagrangians. Let $g=\langle\langle\rangle$,$\rangle be a G$-invariant Riemannian metric on $Q$, for example induced by the kinetic energy of a given mechanical system. Using the given connection $A$, and this metric, we define a new metric (of generalized Kaluza-Klein type) by applying the given metric to the horizontal and vertical components relative to the connection. Specifically, we define the new metric $g_{A}$ by

$$
g_{A}\left(v_{q}, w_{q}\right)=g\left(\operatorname{Hor}_{q} v_{q}, \operatorname{Hor}_{q} w_{q}\right)+g\left(\operatorname{Ver}_{q} v_{q}, \operatorname{Ver}_{q} w_{q}\right) .
$$

Associated with this metric and a given potential energy $V(q)$ is the Kaluza-Klein Lagrangian defined by

$$
L_{A}\left(v_{q}\right)=\frac{1}{2} g_{A}\left(v_{q}, v_{q}\right)-V(q) .
$$

The Mechanical Connection. As above, we assume there is a $G$ invariant metric on the configuration space. Normally this metric is the one associated with the kinetic energy of a given mechanical system. The mechanical connection $A_{0}$ is the connection on $Q$ regarded as a bundle over shape space $Q / G$ that is defined by declaring its horizontal space at a 
point $q \in Q$ to be the subspace that is the orthogonal complement to the tangent space to the group orbit through $q \in Q$ using the kinetic energy metric.

The locked inertia tensor $\mathbb{I}(q): \mathfrak{g} \rightarrow \mathfrak{g}^{*}$ (where $\mathfrak{g}^{*}$ denotes the dual of the vector space $\mathfrak{g}$ ) is defined by

$$
\langle\mathbb{I}(q) \xi, \eta\rangle=\left\langle\left\langle\xi_{Q}(q), \eta_{Q}(q)\right\rangle\right\rangle
$$

where $\xi_{Q}$ is the infinitesimal generator of $\xi \in \mathfrak{g}$ and where $\langle\langle\rangle$,$\rangle is the kinetic$ energy inner product.

An explicit formula for the mechanical connection (see Marsden [1992] for further details and references) is

$$
A_{0}\left(v_{q}\right)=\mathbb{I}(q)^{-1} \mathbf{J}\left(v_{q}\right)
$$

where $\mathbf{J}: T Q \rightarrow \mathfrak{g}^{*}$ is the momentum map defined by

$$
\left\langle\mathbf{J}\left(v_{q}\right), \xi\right\rangle=\left\langle\mathbb{F} L\left(v_{q}\right), \xi_{Q}(q)\right\rangle
$$

where $\mathbb{F} L: T Q \rightarrow T^{*} Q$ is the fiber derivative of $L$.

Another characterization of the mechanical connection is that it picks out the "optimal" rotating frame, i.e., it minimizes the kinetic energy subject to the constraint $\mathbf{J}=\mu$.

The mechanical connection $A_{0}$ plays a fundamental role in the theory of cotangent bundle reduction and amended potentials (going back to work of Smale) as well as in the theory of geometric phases (Marsden, Montgomery and Ratiu [1990]), where holonomy of an associated connection is involved, and in stability theory where it is used to separate internal and rotational modes (Simo, Lewis and Marsden, [1991]).

The mechanical connection has the following physical interpretation for a system of interconnected particles and rigid bodies with a generalized velocity $v_{q}$ at a configuration $q: A_{0}\left(v_{q}\right)$ is the spatial angular velocity of the instantaneously equivalent rigid body system obtained by locking all the joints. Thus, the phrase (spatial) locked angular velocity is sometimes used.

Lines of Connections. Let $A_{0}: T Q \rightarrow \mathfrak{g}$ be a principal $G$-connection on a principal bundle $\pi: Q \rightarrow Q / G$. Recall that a horizontal one-form is one that vanishes on all vertical vectors. If $\tau$ is a $G$-equivariant horizontal $\mathfrak{g}$-valued one form on this bundle, then $A_{\tau}:=A_{0}+\tau$ is also a connection form. We shall denote the corresponding Kaluza-Klein metric by $g_{\tau}$ (so that $g_{\tau}=g_{A_{\tau}}$ as defined earlier) and the associated Lagrangian by $L_{\tau}$. 
In the examples we will be considering, the gain parameter for stabilization can be viewed as parameterizing a point on the line in the space of connections through $A_{0}$, the mechanical connection, and in the direction of $\tau$. Using this family of connections in the Kaluza-Klein Lagrangian produces a family of Lagrangians labeled by the gain parameter.

It will be useful to establish an identity between the free Lagrangian and the Kaluza-Klein Lagrangian $L_{\tau}$. From now on we assume that $A_{0}$ is chosen to be the mechanical connection associated with the free Lagrangian, of the form kinetic energy minus potential, with the kinetic energy given by a $G$-invariant metric $g_{0}$, as above.

Proposition 1.1 For $v \in T_{q} Q$, we have the identity

$$
L_{\tau}(v)=L\left(v+[\tau(v)]_{Q}(q)\right)+\frac{1}{2}\left\|[\tau(v)]_{Q}\right\|^{2}
$$

Proof. This is a consequence of the definition of $L_{\tau}$, the following useful relations between the horizontal and vertical projections for the mechanical and the controlled connections,

$$
\begin{aligned}
& \operatorname{Hor}_{\tau} v=\operatorname{Hor} v-[\tau(v)]_{Q}(q) \\
& \operatorname{Ver}_{\tau} v=\operatorname{Ver} v+[\tau(v)]_{Q}(q),
\end{aligned}
$$

and the fact that in the original metric $\operatorname{Hor}(v)$ is orthogonal to vertical vectors and in particular to $[\tau(v)]_{Q}(q)$. $Q E D$

Another observation that is easily checked is the following.

Proposition 1.2 The mechanical connection associated with the metric $g_{\tau}$ is $A_{\tau}$.

In fact, if one likes, this can be used to characterize the metric $g_{\tau}$ which is used for the kinetic energy in the Lagrangian $L_{\tau}$.

With $A_{0}$ and $\tau$ fixed, one can look at the line in the space of connections through $A_{0}$ in the direction of $\tau$. As we shall see in the examples, moving on this line corresponds to increasing the control gain.

Controlled Lagrangians. In some problems, such as that of stabilizing an inverted pendulum, we need to use a somewhat more general family of Lagrangians than that obtained by the lines of connections construction given above. We will do this by generalizing formula (1.3) as follows. Choose a quadratic form $\sigma_{q}$, a "controlled metric" on each tangent space to the group orbit. In terms of $\sigma$ we make the following. 
Definition 1.3 The controlled Lagrangian $L_{\tau, \sigma}$ associated with the free Lagrangian $L$, the horizontal one form $\tau$, and the metric $\sigma$ is defined by

$$
L_{\tau, \sigma}(v)=L\left(v+[\tau(v)]_{Q}(q)\right)+\frac{1}{2} \sigma_{q}\left([\tau(v)]_{Q}\right) .
$$

As we shall see in the example of the pendulum, we do not want to restrict the form $\sigma$ to be positive definite. Another remark is that choosing $\sigma$ to be the given kinetic energy metric on the fibers (i.e., the locked inertia tensor) gives the Kaluza-Klein Lagrangian in proposition 1.1. One can therefore view the generalization given by the preceding definition as a generalized Kaluza-Klein construction in that the new kinetic energy is the kinetic energy of an identifiable metric, as follows.

Proposition 1.4 The controlled Lagrangian is the kinetic energy function of the (not necessarily positive definite) metric $g_{\tau, \sigma}$ on $Q$ defined as follows. Let $g_{\sigma}$ be the metric which equals $g$ on the space of $A_{0}$-horizontal vectors and which equals $\sigma$ on the vertical vectors (and these two spaces are $g_{\sigma^{-}}$ orthogonal). Define $g_{\tau, \sigma}$ to be $g_{\sigma}$ on the $A_{\tau}$-horizontal vectors and $g$ on the vertical vectors (and these two spaces are $g_{\tau, \sigma}$-orthogonal).

Notice that the controlled Lagrangian $L_{\tau, \sigma}$ is obtained from the original one $L$ by modifying only the kinetic energy. One can also contemplate modifying the potential energy (e.g., by breaking its symmetry) and this will not "interfere" with the construction here. We intend to pursue this point elsewhere.

Below we will develop a strategy for comparing the equations of motion for the "controlled" Lagrangian $L_{\tau}$ and those for the "free" Lagrangian $L_{0}$ so that we can interpret the extra term in the equations of motion as control forces.

The Conservation Law for the Controlled Lagrangian. We now compute the momentum map associated with the controlled Lagrangian $L_{\tau, \sigma}$. This calculation is straightforward using the definition. The resulting momentum map has the form

$$
\mathbf{J}_{\tau, \sigma}=\mathbf{J}+\mathbf{j}_{\tau, \sigma}
$$

where $\mathbf{J}$ is the uncontrolled momentum map (the momentum map for $L$ ) and $\mathbf{j}_{\tau, \sigma}$ is a correction term depending on $\tau$ and $\sigma$. In many examples a formula for $\mathbf{j}_{\tau, \sigma}$ can be computed directly and simply as we shall see, but we give the general formula for completeness. To explain it, first choose a 
basis $e_{a}, a=1, \ldots, \operatorname{dim} \mathfrak{g}$ for the Lie algebra $\mathfrak{g}$ and write elements $\xi \in \mathfrak{g}$ as $\xi=\xi^{a} e_{a}$ (summation understood) and choose coordinates $q^{i}, i=1, \ldots, n$ on $Q$. The dual basis of $\mathfrak{g}^{*}$ is denoted $e^{a}$ and elements of $\mathfrak{g}^{*}$ are written $\mu=\mu_{a} e^{a}$. The standard momentum map associated with a Lagrangian $L$ is written $\mathbf{J}=J_{a} e^{a}$ and is given in coordinates by

$$
J_{a}=\frac{\partial L}{\partial \dot{q}^{i}} K_{a}^{i},
$$

where $K_{a}^{i}$ are the action coefficients defined by writing the infinitesimal generator $[\eta]_{Q}$ of a Lie algebra element $\eta \in \mathfrak{g}$ as $[\eta]_{Q}^{i}(q)=K_{a}^{i}(q) \eta^{a}$.

We now give the formula for $\mathbf{j}_{\tau, \sigma}$, which is the correction terms in the momentum map $\mathbf{J}_{\tau, \sigma}$ for the Lagrangian $L_{\tau, \sigma}$ verses the momentum map $\mathbf{J}$ for $L$. We first give the formula in coordinates, writing, as with $\mathbf{J}$, the components of $\mathbf{j}_{\tau, \sigma}$ as $j_{a}$. We have

$$
j_{a}=K_{a}^{i} \tau_{i}^{b}\left(J_{b}+\sigma_{c b} \tau_{j}^{c} \dot{q}^{j}\right),
$$

where $\sigma_{c b}$ are the components of the tensor $\sigma$ but thought of as a configuration dependent bilinear form on the Lie algebra and $\tau_{j}^{a}$ are the components of $\tau$ thought of as a Lie algebra valued one form.

Intrinsically, the formula for $\mathbf{j}_{\tau, \sigma}$ may be written as follows at a point $v_{q} \in T_{q} Q:$

$$
\mathbf{j}_{\tau, \sigma}\left(v_{q}\right)=\left(\psi_{q}^{*} \circ \tau_{q}^{*}\right)\left(\mathbf{J}\left(v_{q}\right)+\tau\left(v_{q}\right)^{b}\right),
$$

where

$$
\psi_{q}: \mathfrak{g} \rightarrow T_{q} Q ; \xi \rightarrow[\xi]_{Q}(q)
$$

is the infinitesimal generator map, $\tau_{q}: T_{q} Q \rightarrow \mathfrak{g}$ is the restriction of $\tau$ to the fiber over $q$, the star denotes the dual linear transformation and where the flat denotes the corresponding covector in $\mathfrak{g}^{*}$ using the bilinear form $\sigma$.

Since the controlled Lagrangian is group invariant, we get, by Noether's theorem, the conservation law

$$
\frac{d}{d t}\left(\mathbf{J}+\mathbf{j}_{\tau, \sigma}\right)=0
$$

which, rewriting as

$$
\frac{d}{d t} \mathbf{J}=u
$$

defines the control force in the symmetry direction. For the case of the satellite with the internal rotor, the symmetry direction in question will be the rotor angle and so this indeed corresponds to what we want, namely the control force on the rotor. For the case of the pendulum on the cart, the symmetry direction will be the direction of the cart and so $u$ will be the force on the cart. 
Relative Equilibria. Since $\tau$ is horizontal, for any Lie algebra element $\xi \in \mathfrak{g}$, we have $\tau\left(\xi_{Q}(q)\right)=0$ and so we have the identity $L\left(\xi_{Q}(q)\right)=$ $L_{\tau, \sigma}\left(\xi_{Q}(q)\right)$; that is, $L$ and $L_{\tau, \sigma}$ have the same locked Lagrangian. From the fact that relative equilibria are the critical points of the locked Lagrangian (see Lewis [1992], Prop 2.3) we conclude the following:

Proposition 1.5 The relative equilibria for $L$ and $L_{\tau, \sigma}$ are the same.

Of course one can now use the energy momentum method (Simo, Lewis, and Marsden [1991], Marsden [1992]), especially its Lagrangian formulation (Lewis [1992], Wang and Krishnaprasad [1992]) to ascertain stability. Development of this idea will be the subject of future investigations.

The General Strategy. Our constructions produce a $G$-invariant Lagrangian provided that the data $L, \tau$ and $\sigma$ are $G$-invariant (or, as appropriate, equivariant). This will be the case in both of our examples. In this situation, one can interpret the new equation for the old momentum map as defining a control force in the symmetry direction as was explained above.

We summarize the overall situation and strategy so far as follows.

- Start with a mechanical system with a Lagrangian $L$ of the form kinetic minus potential energy and a symmetry group $G$.

- Introduce a horizontal one form $\tau$ on $Q$ (it vanishes in the group directions) and a quadratic form $\sigma$ on each tangent space to the group orbits and define the controlled Lagrangian by

$$
L_{\tau, \sigma}(v)=L\left(v+[\tau(v)]_{Q}(q)\right)+\frac{1}{2} \sigma_{q}\left([\tau(v)]_{Q}\right) .
$$

- Compute the control force in the symmetry direction using Noether's theorem for the controlled Lagrangian, as in equations (1.9) and (1.10).

- The quantities $\tau$ and $\sigma$ are chosen so that the controlled EulerLagrange equations for the original system (i.e., the Euler-Lagrange equations for the Lagrangian $L$ with an control) agree with the EulerLagrange equations for the controlled Lagrangian $L_{\tau, \sigma}$. We determine a feedback law for $u$, by using the Euler-Lagrange equations to eliminate accelerations; then the control law becomes a feedback that is configuration and, possibly, velocity dependent. 
- This method is effective for determining stabilizing feedback laws. The stability of an equilibrium may be determined by the energymomentum (or energy-Casimir-Arnold) method, using any available freedom in the choice of $\tau$ and $\sigma$.

Lagrangian Reduction. Our constructions are intended to be viewed on the original configuration space (or its tangent bundle), including any configuration variables needed for control actuation. However, if one is keeping the symmetry as we have indicated, one can make use of the theory of Lagrangian reduction to obtain reduced Euler-Lagrange equations in fewer configuration variables (see Marsden and Scheurle [1993a,b] and Bloch, Krisnaprasad, Marsden and Murray [1996]). Thus, one may also compare the equations for the controlled system and the controlled Lagrangian in reduced form. In the example of the rigid body, discussed below, we will be implicitly doing this, but will not do it explicitly for the pendulum on a cart although the reduction procedure is very simple in that case. In particular, this reduction process recovers the observations in Bloch, Krishnaprasad, Marsden and Sánchez de Alvarez [1992] concerning Lie-Poisson (and Euler-Poincaré) structures for the controlled system.

One of the important ingredients in the reduced Euler-Lagrange equations is the curvature of the connection. In this regard, we note that the curvature of the connection $A_{\tau}$ is given by (see Bloch, Krishnaprasad, Marsden and Murray [1996, §3.3]):

$$
\mathcal{B}_{\tau}=\mathbf{d} A-\left[A_{\tau}, A_{\tau}\right]=\mathcal{B}_{0}+\mathbf{d} \tau+\left[A_{0}, \tau\right]+[\tau, \tau] .
$$

\section{The Inverted Pendulum on a Cart.}

In this section we show how the ideas above can be applied to the problem of the inverted pendulum on a cart. This example we hope will show the effectiveness of the methods for the stabilization of balance systems. Related examples we have in mind are systems like the inverted pendulum on a hockey puck (which we plan to study in a future publication) and the bicycle (see, for example, Getz and Marsden [1994] and Koon and Marsden [1996]).

The Lagrangian for the cart-pendulum system. Let $s$ denote the position of the cart on the $s$-axis and let $\theta$ denote the angle of the pendulum from the upright vertical, as in figure 1. 


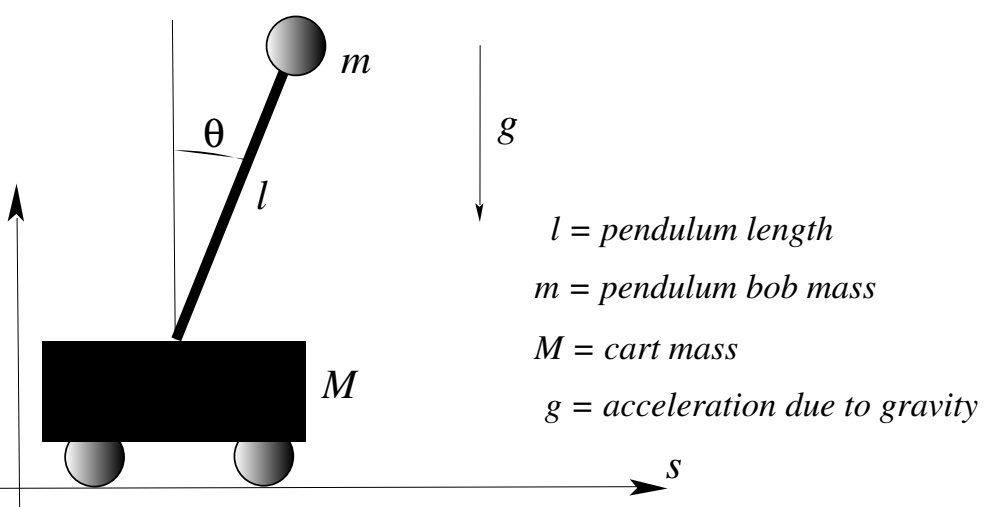

Figure 1: The pendulum on a cart system

Here the configuration space is $Q=\mathbb{R} \times S^{1}$ with the first factor being the cart position $s$, and the second factor being the pendulum angle, $\theta$. The velocity phase space, $T Q$ has coordinates $(s, \theta, \dot{s}, \dot{\theta})$.

The velocity of the cart relative to the lab frame is of course $\dot{s}$, while the velocity of the pendulum relative to the lab frame is the vector

$$
v_{\text {pend }}=(\dot{s}+l \cos \theta \dot{\theta},-l \sin \theta \dot{\theta}) .
$$

The system kinetic energy is just the sum of the kinetic energies of the cart and the pendulum:

$$
K\left((s, \theta, \dot{s}, \dot{\theta})=\frac{1}{2}(\dot{s}, \dot{\theta})\left(\begin{array}{cc}
M+m & m l \cos \theta \\
m l \cos \theta & m l^{2}
\end{array}\right)\left(\begin{array}{c}
\dot{s} \\
\dot{\theta}
\end{array}\right) .\right.
$$

The Lagrangian is the kinetic minus potential energies, so we get

$$
L(s, \theta, \dot{s}, \dot{\theta})=K(s, \theta, \dot{s}, \dot{\theta})-V(\theta),
$$

where the potential energy is $V=m g l \cos \theta$.

The symmetry group $G$ of the pendulum-cart system is that of translation in the $s$ variable so $G=\mathbb{R}$. We do not destroy this symmetry when doing stabilization in $\theta$; we would, however, use symmetry breaking potentials to track in the variable $s$ if tracking were our goal. For the moment we are focusing on stabilizing this balance system.

The Linearized Lagrangian. We specialize to the case of the linearized Lagrangian about the upright position of the pendulum. We do this for sim- 
plicity of exposition; the method is fully nonlinear and does not depend on linearization. With a few modifications, as indicated below, the techniques we develop here work perfectly well for the full nonlinear pendulum and for many other examples as well.

The linearized Lagrangian is

$$
L(s, \theta, \dot{s}, \dot{\theta})=\frac{1}{2}\left(\alpha \dot{\theta}^{2}+2 \beta \dot{s} \dot{\theta}+\gamma \dot{s}^{2}\right)-\frac{1}{2} D \theta^{2},
$$

where $\alpha=m l^{2}, \beta=m l, \gamma=M+m$ and $D=-m g l$. Positive definiteness of the mass matrix corresponds to the inequality $\alpha \gamma-\beta^{2}>0$.

Notice that the momentum conjugate to $s$ is $p_{s}=\gamma \dot{s}+\beta \dot{\theta}$ and that the momentum conjugate to $\theta$ is $p_{\theta}=\alpha \dot{\theta}+\beta \dot{s}$.

The relative equilibrium defined by $\theta=0, \dot{\theta}=0$ and $\dot{s}=0$ is unstable since $D<0$.

The Controlled Cart. The equations of motion of the cart pendulum system with a control force $u$ acting on the cart (and no direct forces acting on the pendulum) are, since $s$ is a cyclic variable,

$$
\begin{aligned}
\frac{d}{d t} \frac{\partial L}{\partial \dot{s}} & =u \\
\frac{d}{d t} \frac{\partial L}{\partial \dot{\theta}}-\frac{\partial L}{\partial \theta} & =0
\end{aligned}
$$

i.e.,

$$
\begin{aligned}
\frac{d}{d t} p_{s} & =\frac{d}{d t}(\gamma \dot{s}+\beta \dot{\theta})=u \\
\frac{d}{d t} p_{\theta}+D \theta & =\frac{d}{d t}(\alpha \dot{\theta}+\beta \dot{s})+D \theta=0
\end{aligned}
$$

The Controlled Lagrangian. Recall that we form the controlled Lagrangian by modifying only the kinetic energy of the free pendulum cart Lagrangian according to (1.4), which involves the choice of $\tau$ and $\sigma$.

In this case, clearly any horizontal one form $\tau$ is a multiple of $d \theta$ and $\sigma$ is just a scalar. Since we are dealing with the linearized system, $\sigma$ is not configuration space dependent. Thus, let $\sigma$ be a number and define the one form $\tau=k d \theta$. (For the full nonlinear pendulum one should take $k$ to be a function of $\theta$; specifically the choice $k(\theta)=\kappa \cos \theta$ will do.) Following the general construction, we let

$$
L_{\tau, \sigma}:=\frac{1}{2}\left(\alpha \dot{\theta}^{2}+2 \beta(\dot{s}+k \dot{\theta}) \dot{\theta}+\gamma(\dot{s}+k \dot{\theta})^{2}\right)+\frac{\sigma}{2} \gamma k^{2} \dot{\theta}^{2}-\frac{1}{2} D \theta^{2} .
$$


Notice also that, as in the general theory, the variable $\dot{s}$ is "shifted" and a term quadratic in $\dot{\theta}$ is added.

Notice that the variable $s$ is still cyclic. Following the guidelines of the theory, we look for the feedback control by looking at the change in the conservation law. Associated to the new Lagrangian $L_{\tau, \sigma}$, we have the associated conservation law

$$
\frac{d}{d t}(\beta \dot{\theta}+\gamma(\dot{s}+k \dot{\theta}))=0
$$

which we can rewrite using the same conjugate momentum $p_{s}$ as for the uncontrolled Lagrangian as follows

$$
\frac{d}{d t} p_{s}=u:=-\gamma k \ddot{\theta}
$$

Thus, we identify the term on the right hand side as the control force exerted on the cart.

Still using the controlled Lagrangian, as well as (2.6), the $\theta$ equation is computed to be

$$
\left(\alpha-\frac{\beta^{2}}{\gamma}+\gamma k^{2} \sigma\right) \ddot{\theta}+D \theta=0 .
$$

For many examples, such as the the rigid body with an internal rotor, it is important here to use the conservation law itself rather than its differentiated form.

Interestingly, the Euler-Lagrange equation for $\theta$ using the controlled Lagrangian agrees with the $\theta$ equation for the controlled cart provided $\sigma=-\beta /(\gamma k)$, as a direct calculation shows. (The choice $\sigma=-\beta /(\gamma \kappa)$ is used for the nonlinear pendulum).

The Cart Feedback Control. By manipulating the preceding EulerLagrange equations for the controlled Lagrangian, we obtain the resulting control law:

$$
u=\nu \theta
$$

where

$$
\nu=\frac{\gamma^{2} k D}{\left(\alpha \gamma-\beta^{2}+\gamma^{2} k^{2} \sigma\right)}=\frac{\gamma^{2} k D}{\alpha \gamma-\beta^{2}-\gamma k \beta} .
$$

Stabilization. Since this system is so simple, stabilization can be readily analyzed using the dynamics of a harmonic oscillator. Since $D<0$, the 
$\theta$ dynamics is stabilized if the coefficient of $\ddot{\theta}$ is negative, which gives the condition

$$
\alpha \gamma-\beta^{2}-\gamma k \beta<0
$$

Simplifying, this stability condition becomes

$$
k>\frac{\alpha \gamma-\beta^{2}}{\beta \gamma}>0 .
$$

Thus, $k$ is positive and in this case, $\nu>0$, which is the correct sign for a stabilizing proportional position controller.

In summary, we get a stabilizing proportional feedback control law provided $k$ is chosen to be positive and it satisfies the inequality (2.10).

This approach is nice because it is done within the context of mechanics; one can understand the stabilization in terms of the effective creation of an energy well by the feedback control.

\section{The rigid body with a symmetric rotor.}

Following Krishnaprasad [1985] and Bloch, Krishnaprasad, Marsden and Sánchez de Alvarez [1992], we consider a rigid body with a rotor aligned along the third principal axis of the body; see Figure 2. The rotor spins under the influence of a torque $u$ acting on the rotor. The Lagrangian (discussed explicitly below) is simply the total kinetic energy of the system, rigid carrier plus the rotor kinetic energy, with no potential energy.

Equations of Motion. The equations of motion are given by

$$
\begin{aligned}
\dot{\Pi} & =\Pi \times \Omega \\
i & =u,
\end{aligned}
$$

where

- $I_{1}>I_{2}>I_{3}$ are the rigid body moments of inertia,

- $J_{1}=J_{2}$ and $J_{3}$ are the rotor moments of inertia.

- $\Omega=\left(\Omega_{1}, \Omega_{2}, \Omega_{3}\right)$ is the body angular velocity vector of the carrier

- $\alpha$ is the relative angle of the rotor. 


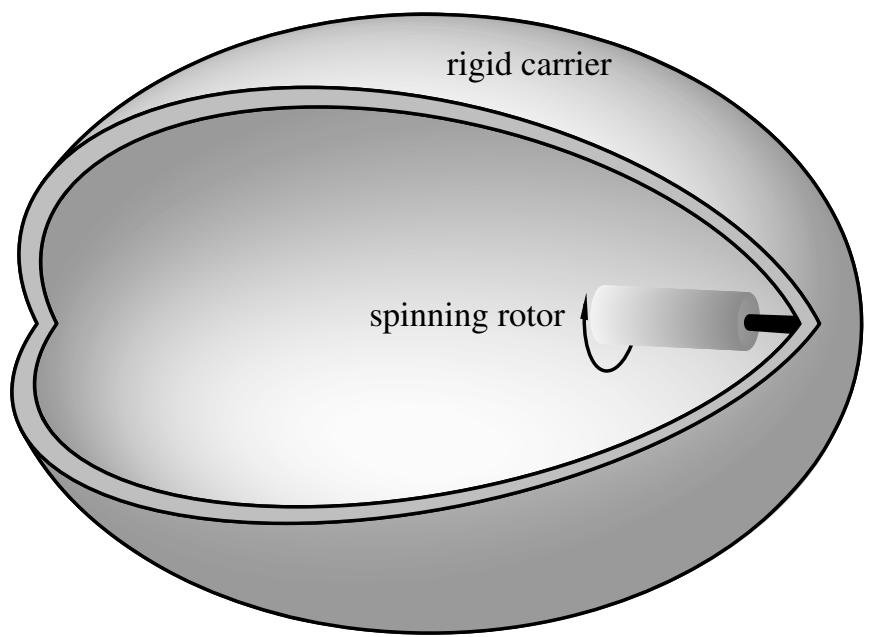

Figure 2: A rigid body with a rotor aligned on the long axis

- The body angular momenta are determined by the Legendre transform to be

$$
\begin{aligned}
\Pi_{1} & =\lambda_{1} \Omega_{1} \\
\Pi_{2} & =\lambda_{2} \Omega_{2} \\
\Pi_{3} & =\lambda_{3} \Omega_{3}+J_{3} \dot{\alpha} \\
l_{3} & =J_{3}\left(\Omega_{3}+\dot{\alpha}\right),
\end{aligned}
$$

where $\lambda_{i}=I_{i}+J_{i}$.

The equations written out in components relative to a principal axis frame are

$$
\begin{aligned}
\dot{\Pi}_{1} & =\left(\frac{1}{I_{3}}-\frac{1}{\lambda_{2}}\right) \Pi_{2} \Pi_{3}-\frac{l_{3} \Pi_{2}}{I_{3}} \\
\dot{\Pi}_{2} & =\left(\frac{1}{\lambda_{1}}-\frac{1}{I_{3}}\right) \Pi_{1} \Pi_{3}+\frac{l_{3} \Pi_{1}}{I_{3}} \\
\dot{\Pi}_{3} & =\left(\frac{1}{\lambda_{2}}-\frac{1}{\lambda_{1}}\right) \Pi_{1} \Pi_{2} \\
\dot{l}_{3} & =u .
\end{aligned}
$$

Although we shall work with the reduced equations as we have stated them, it is important to keep in mind that these equations may be regarded 
as coming from a configuration space via either Hamiltonian or Lagrangian reduction. The original or primitive configuration space is $Q=\mathrm{SO}(3) \times S^{1}$, corresponding to the rigid carrier and the rotor variables. There are two commuting symmetry groups in the problem, namely the left action of $\mathrm{SO}(3)$ and the right action of $S^{1}$. For the constructions in this paper, the symmetry group is regarded as $G=S^{1}$, but we are also freely reducing by the commuting group $K=\mathrm{SO}(3)$, as is convenient. It is clear that for our general constructions that one can pass the ideas through a commuting symmetry group reduction.

The Feedback Control and Hamiltonian Structure. If the control force is zero, i.e., $u=0$, then the $S^{1}$ symmetry of the rotor gives the obvious conservation law, namely that $l_{3}$ is a constant of motion. Substituting this conservation law into the remaining equations for the body angular momentum, one finds that they are Hamiltonian with respect to the LiePoisson bracket and with Hamiltonian function

$$
H=\frac{1}{2}\left(\frac{\Pi_{1}^{2}}{\lambda_{1}}+\frac{\Pi_{2}^{2}}{\lambda^{2}}+\frac{\left(\Pi_{3}-l_{3}\right)^{2}}{I_{3}}\right)+\frac{1}{2} l_{3}^{2} .
$$

The Lie-Poisson bracket used here is the standard one for $\mathfrak{s o}(3)^{*}$, namely the rigid body bracket. (See Marsden and Ratiu [1994] for general background.) This Lie-Poisson structure follows by general properties of cotangent bundle and Lie-Poisson reduction.

The feedback control law chosen in Bloch, Krishnaprasad, Marsden and Sánchez de Alvarez [1992] is given by

$$
u=k\left(\frac{1}{\lambda_{2}}-\frac{1}{\lambda_{1}}\right) \Pi_{1} \Pi_{2},
$$

where $k$ is a gain parameter.

With this feedback law, notice that the system retains the $S^{1}$ symmetry and has, by direct calculation, a new conserved quantity given by $P_{k}=$ $l_{3}-k \Pi_{3}$.

Eliminating the rotor variable using this conservation law leads to the closed loop equations

$$
\begin{aligned}
\dot{\Pi}_{1} & =\Pi_{2}\left(\frac{(1-k) \Pi_{3}-P_{k}}{I_{3}}\right)-\frac{\Pi_{3} \Pi_{2}}{\lambda_{2}} \\
\dot{\Pi}_{2} & =-\Pi_{1}\left(\frac{(1-k) \Pi_{3}-P_{k}}{I_{3}}\right)+\frac{\Pi_{1} \Pi_{3}}{\lambda_{1}} \\
\dot{\Pi}_{3} & =\left(\frac{1}{\lambda_{2}}-\frac{1}{\lambda_{1}}\right) \Pi_{1} \Pi_{2} .
\end{aligned}
$$


Noteworthy special cases are

1. $k=0$, the uncontrolled case,

2. $k=J_{3} / \lambda_{3}$, the driven case where $\dot{\alpha}=$ constant, so the rotors are driven with constant angular velocity.

The preceding displayed equations are also Hamiltonian with

$$
H=\frac{1}{2}\left(\frac{\Pi_{1}^{2}}{\lambda_{1}}+\frac{\Pi_{2}^{2}}{\lambda_{2}}+\frac{\left((1-k) \Pi_{3}-P_{k}\right)^{2}}{(1-k) I_{3}}\right)+\frac{1}{2} \frac{P_{k}^{2}}{J_{3}(1-k)},
$$

again using the Lie-Poisson (rigid body) Poisson structure on $s o(3)^{*}$. This may be verified by a direct calculation, although the reasons for such a structure may seem mysterious at this point. After all, the rotor is being forced (work is being done!) and so why should the resulting equations be Hamiltonian at all? Of course, there is no immediate contradiction since the Hamiltonian is not simply the system kinetic energy. As we shall see, this structure fits into the general scheme of the present paper.

How the Rigid Body with a Rotor fits the General Scheme. We start with the free Lagrangian given, as mentioned, by the system kinetic energy. We write it in reduced form, but it may be equally well regarded as being defined on $T Q$. It is given by

$$
L_{0}=\frac{1}{2}\left(\lambda_{1} \Omega_{1}^{2}+\lambda_{2} \Omega_{2}^{2}\right)+\frac{1}{2} I_{3} \Omega_{3}^{2}+\frac{1}{2} J_{3}\left(\Omega_{3}+\dot{\alpha}\right)^{2} .
$$

Recall that the Euler-Poincaré equations for a Lagrangian $l$ on a Lie algebra $\mathfrak{g}$ are given in coordinates by

$$
\frac{d}{d t} \frac{\partial l}{\partial \xi^{d}}=C_{a d}^{b} \frac{\partial l}{\partial \xi^{b}} \xi^{a}
$$

where $C_{a d}^{b}$ are the structure constants of the Lie algebra relative to a given basis of $\mathfrak{g}$. See Marsden and Ratiu [1994] for a general discussion of these equations and for their intrinsic formulation.

The equations (3.1) with $u=0$ (and thought of as functions of the body angular velocities) are readily checked to be the Euler-Poincaré equations for the Lagrangian $L_{0}$ on the Lie algebra $\mathfrak{g}=\mathfrak{s o}(3) \times \mathbb{R}$. In the closed loop case, we likewise get Euler-Poincaré equations on $\mathfrak{s o}(3)$.

As we also mentioned, the conserved quantity associated with the $S^{1}$ action (the symmetry of the rotor) is

$$
P_{0}=J_{3}\left(\Omega_{3}+\dot{\alpha}\right)=l_{3} .
$$


We choose $\sigma$ to be the metric determined by $L_{0}$; that is, we are going to be in the pure Kaluza-Klein case in this example. Since our group is $S^{1}$, any ( $\mathrm{SO}(3)$-equivariant) horizontal one form must be a linear combination of the carrier angular velocities (thinking of this as a one-form). We choose it to be a multiple of $\Omega_{3}$. The particular multiple is chosen so that the resulting control force is of the form given in equation (3.2). Namely, we write

$$
\tau=-\frac{k}{1-k} \frac{I_{3}}{J_{3}} \Omega_{3}:=r \Omega_{3},
$$

which defines $r$. We also choose $\sigma$ to be the standard kinetic energy metric. Construct, according to the general procedure, a new Lagrangian obtained by replacing $\dot{\alpha}$ by $\dot{\alpha}+\tau_{Q}$ and adding $\frac{1}{2}\left\|\tau_{Q}\right\|^{2}$; one gets

$$
L_{\tau, \sigma}=\frac{1}{2}\left(\lambda_{1} \Omega_{1}^{2}+\lambda_{2} \Omega_{2}^{2}\right)+\frac{1}{2} I_{3} \Omega_{3}^{2}+\frac{1}{2} J_{3}\left((1+r) \Omega_{3}+\dot{\alpha}\right)^{2}+\frac{1}{2} J_{3}\left(r \Omega_{3}\right)^{2} .
$$

Amazingly enough, one computes that the momentum conjugate to $\alpha$ for this Lagrangian is $P_{k}$ (up to a factor of $1-k$ ) and the resulting Euler-Poincaré equations give the feedback controlled system! Thus, our construction explains the otherwise "strange" Lagrangian and Hamiltonian structures.

Stabilization. Once one has the problem in Lagrangian and hence Hamiltonian form, one can proceed to use the energy-Casimir or energymomentum method to determine stability. This is often much more computationally efficient than an analysis of eigenvalues of the linearized equations (which, in any case, need not imply nonlinear stability in the mechanical case).

As in Bloch, Krishnaprasad, Marsden and Sánchez de Alvarez [1992], we consider the case $P=0$ and the special equilibrium $(0, M, 0)$. The energy-Casimir method then shows that

Proposition 3.1 For $k>1-J_{3} / \lambda_{2}$, the equilibrium $(0, M, 0)$ is nonlinearly stable.

Indeed, we look at $H+C$ where $C=\varphi\left(\|m\|^{2}\right)$. Pick $\varphi$ so that the first variation vanishes:

$$
\left.\delta(H+C)\right|_{(0, M, 0)}=0,
$$

One computes that $\delta^{2}(H+C)$ is negative definite if $k>1-J_{3} / \lambda_{2}$ and $\varphi^{\prime \prime}\left(M^{2}\right)<0$, which proves the claim.

The stabilization that takes place as the gain is increased can be viewed in terms of a modification of the phase portrait of the rigid body: the four 
heteroclinic orbits for the rigid body close up along the "hinge" joining the two saddle points forming a circle of fixed points and then open up along a "hinge" joining two stable points, forming a stability island where there were saddle points previously.

The feedback control in effect modifies the Lagrangian to interchange the moments of inertia of the system.

Other related examples can be treated in a similar way. For example, one can use these techniques to stabilize a spinning Lagrange top (a heavy top with a fixed point rotating in a gravitational field) using a torque control on a rotor attached along the symmetry axis of the top.

\section{Concluding Remarks.}

As we have indicated, the technique in this paper can be combined with the technique of Leonard [1996] who introduced symmetry breaking potentials for purposes of stabilizing relative equilibria of underwater vehicles. (The potentials can be for either rotational or translational symmetry breaking). The nice thing is that the two methods can simply be concatenated; if the "balance stability" has been achieved in some of the variables (here the carrier angular velocity variables), then the symmetry breaking potentials can be introduced by additional control forces that do not destroy the achieved stability.

It is also expected that one can extend the techniques for purposes of tracking by forming a tracking function $\mathcal{T}(t)$ by taking the function produced by the energy-Casimir method, but with the relative equilibrium (which is a minimum of the function) replaced by the trajectory one wishes to track. The fact that this trajectory is time dependent introduces an explicit time dependence into the tracking function. One then computes the total time derivative of $\mathcal{T}(t)$ under the influence of control forces and requires that these control forces decrease $\mathcal{T}(t)$; thus, one is guaranteed of tracking, perhaps approximately, its minimum. For a relative equilibrium obviously no control forces are needed. The tracking methodology suggested by this approach is in the spirit of that of Koditschek and Rimon [1990].

\section{Acknowledgments.}

We thank John Ballieul, Francesco Bullo, Joel Burdick, Neil Getz, Dan Koditschek, P.S. Krishnaprasad, Naomi Leonard, Richard Murray and Tudor Ratiu for helpful comments. Some of the ideas here were directly in- 
spired by our efforts to combine ideas in Bloch, Krishnaprasad Marsden and Sánchez de Alvarez [1992], those of Wang and Krishnaprasad [1992], and those in the $\mathrm{PhD}$ thesis of Neil Getz.

\section{References}

Ballieul, J. [1993] Stable average motions of mechanical systems subject to periodic forcing. Fields Inst. Comm. 1, 1-23.

Bloch, A.M., P.S. Krishnaprasad, J.E. Marsden, and R. Murray [1996] Nonholonomic mechanical systems with symmetry. Arch. Rat. Mech. An. (to appear).

Bloch, A.M., P.S. Krishnaprasad, J.E. Marsden, and T.S. Ratiu [1996] The Euler-Poincaré equations and double bracket dissipation. Comm. Math. Phys. 175, 1-42.

Bloch, A.M., P.S. Krishnaprasad, J.E. Marsden and G. Sánchez de Alvarez [1992] Stabilization of rigid body dynamics by internal and external torques. Automatica 28, 745-756.

Bloch, A.M., M. Reyhanoglu and H. McClamroch [1992] Control and stabilization of nonholonomic systems. IEEE Trans. Aut. Control 37, 1746-1757.

Getz, N.H. and J.E. Marsden [1995] Control for an autonomous bicycle, International Conference on Robotics and Automation, IEEE, Nagoya, Japan, May, 1995.

Koditschek, D.E. [1989] The application of total energy as a Lyapunov function for mechanical control systems, in Dynamics and control of multibody systems (Brunswick, ME, 1988), 131-157, Contemp. Math., 97, Amer. Math. Soc., Providence, RI.

Koditschek, D.E. and E. Rimon [1990] Robot navigation functions on manifolds with boundary. Adv. in Appl. Math. 11, 412-442.

Koon, W.S. and J.E. Marsden [1996] The Hamiltonian and Lagrangian approaches to nonholonomic mechanics Caltech preprint.

Krishnaprasad, P.S. [1985] Lie-Poisson structures, dual-spin spacecraft and asymptotic stability, Nonl. Anal. Th. Meth. and Appl. 9, 1011-1035. 
Leonard, N.E. [1995] Stability of a bottom-heavy underwater vehicle, $\mathrm{Me}$ chanical and Aerospace Engineering, Princeton University Technical Report 2048, to appear in Automatica 33, March 1997.

Leonard, N.E. [1996] Stabilization of underwater vehicle dynamics with symmetry breaking potentials, in preparation. (See also Leonard, N.E., Stabilization of steady motions of an underwater vehicle, Proc. 35th IEEE Conference on Decision and Control, Kobe, Japan, 1996.)

Leonard, N.E. and J.E. Marsden [1996] Stability and Drift at Nongeneric Momenta and Underwater Vehicle Dynamics, Mechanical and Aerospace Engineering, Princeton University Technical Report 2075,

Lewis, D. [1992] Lagrangian block diagonalization. Dyn. Diff. Eqn's. 4 $1-42$.

Marsden, J.E. [1992], Lectures on Mechanics London Mathematical Society Lecture note series. 174, Cambridge University Press.

Marsden, J.E., R. Montgomery and T.S. Ratiu [1990] Reduction, symmetry, and phases in mechanics. Memoirs AMS 436.

Marsden, J.E. and T.S. Ratiu [1994] Symmetry and Mechanics. Texts in Applied Mathematics, 17, Springer-Verlag.

Marsden, J.E. and J. Scheurle [1993a] Lagrangian reduction and the double spherical pendulum, ZAMP 44, 17-43.

Marsden, J.E. and J. Scheurle [1993b] The reduced Euler-Lagrange equations, Fields Institute Comm. 1, 139-164.

Sánchez de Alvarez, G. [1989] Controllability of Poisson control systems with symmetry, Cont. Math. AMS 97, 399-412.

Simo, J.C., D.R. Lewis and J.E. Marsden [1991] Stability of relative equilibria I: The reduced energy momentum method. Arch. Rat. Mech. Anal. 115, 15-59.

Wang, L.S. and P.S. Krishnaprasad [1992] Gyroscopic control and stabilization, J. Nonlinear Sci. 2, 367-415. 
Department of Mathematics

University of Michigan

Ann Arbor, MI 48109

abloch@math.Isa.umich.edu

Control and Dynamical Systems 116-81

California Institute of Technology

Pasadena, CA 91125

marsden@cds.caltech.edu

Departamento de Matemáticas

Facultad de Ciencias

Universidad Los Andes

Mérida, Venezuela

sanchezg@ciens.ula.ve

Received September, 1996 